\title{
Kinematic and chemical analysis of PNe in NGC 3109
}

\author{
Sheila N. Flores-Dúran ${ }^{1}$, Miriam Peña ${ }^{1}$ and María T. Ruiz ${ }^{2}$ \\ ${ }^{1}$ Instituto de Astronomía, Universidad Nacional Autónoma de México, \\ Apdo. Postal 70264, Ciudad de México, 04510, México \\ email: sflores@astro.unam.mx \\ ${ }^{2}$ Depto. de Astronomía, Universidad de Chile, Casilla 36D, Las Condes, Santiago, Chile
}

\begin{abstract}
We present high resolution spectroscopy obtained with MIKE-Magellan and MES OAN-SPM of a number of planetary nebulae (PNe) and $\mathrm{H}$ II regions, distributed along the dwarf irregular galaxy NGC 3109 and compare their kinematical behavior with the one of H I data. We aim to determine if there is a kinematical connection among these objects. We also perform a revision of the chemical composition of PNe and $\mathrm{H}$ II regions in this galaxy and discuss it in comparison with stellar evolution models.
\end{abstract}

Keywords. galaxies individual: NGC 3109, galaxies: kinematics ans dynamics, ISM abundances: $\mathrm{H}$ II regions and Planetary Nebulae.

\section{Introduction}

The kinematics of Planetary Nebulae $(\mathrm{PNe})$ in galaxies is a clue to understand the behavior of low-intermediate mass stars (LIMS) and their relation with other components of the galaxies.

NGC 3109 is a Magellanic-type irregular galaxy, classified as a late-type spiral SB(s)m. It is one of the most metal-poor galaxies in the vicinity of the Local Group and contains a H I rotating disk (Ott et al. 2012).

Peña et al. (2007a) reported the discovery of twenty PNe in NGC 3109 and Peña et al. (2007b) analyzed the chemistry of some of them finding that the oxygen abundance in $\mathrm{PNe}$ is systematically higher than in $\mathrm{H}$ II regions.

\section{Overview}

The heliocentric radial velocities (RV) of the objects were compared to the velocities of the H I disk at the same projected position. Physical conditions and ionic abundances of PNe and HiI regions in NGC 3109, were obtained from the emission lines given by Peña et al. (2007b). Delgado-Inglada et al. (2014) ionization correction factors were used to derive the total chemical abundances.

The radial velocities (RV) were measured with a precision better than $7-8 \mathrm{~km} \mathrm{~s}^{-1}$. Fig. 1 shows velocities of objects (relative to the systemic velocity of $404 \mathrm{~km} / \mathrm{s}$ ) vs. their position projected along the major axis of the $\mathrm{H}$ I disk. It can be seen that H II regions (open black squares) follow closely the rotation of the H I disk but with a large dispersion. The PNe (black dots) also rotate in the same direction that the disk. We have included RV given by Evans et al. (2007) for luminous blue supergiant stars (green asterisks). These relatively young objects, show a rotation similar to the H I rotation but with a large dispersion, even larger than that presented by our PN sample. 
Figure 1

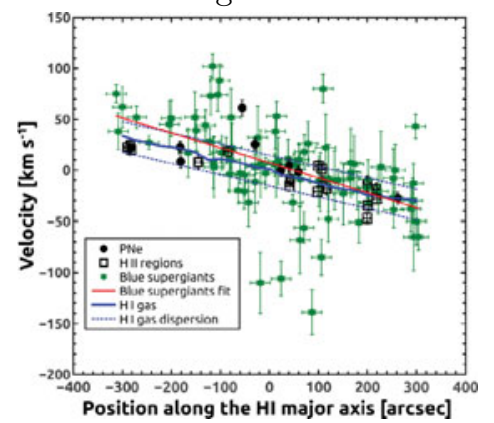

Figure 2

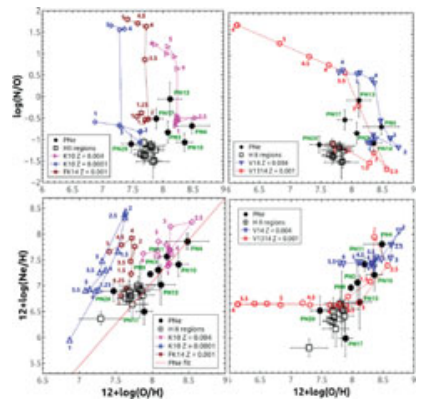

From the chemical abundance determinations we confirm that PNe are enriched in oxygen as Peña et al. (2007b) previously found. The oxygen abundance for H in regions is $\log O / H+12=7.76 \pm 0.07$ and $\mathrm{PNe}$ show, in average, significantly higher oxygen abundance $\log O / H+12=8.17 \pm 0.17$. Similarly to what has been suggested for some of the PNe in the Magellanic Clouds and other metal-poor galaxies, we argue that oxygen in the PNe in NGC 3109 is affected by the third dredge-up event in their progenitors. This also occurs for neon, as we notice that neon is also enriched in PNe, as predicted by stellar evolution models for a $Z=0.004$ (Karakas 2010, K10; Ventura et al. (2013, 2014b, V1314) and Ventura et al. (2014a, V14)), see Fig. 2. We found one confirmed Peimbert Type I PN.

\section{Results}

We found that H II regions share the H I gas kinematics, with differences in velocities smaller than $15 \mathrm{~km} \mathrm{~s}^{-1}$. On the other hand, PNe and blue supergiant stars, even when they rotate in the same direction, show a large dispersion in velocities. This could be indicating that these young-intermediate age objects (blue supergiant stars and $\mathrm{PNe}$ ) belong to the bar that has been reported for the stellar component of NGC 3109.

The PNe in NGC 3109 are oxygen overabundant with respect to the H II regions (0.41 dex). In average Ne enrichment is also seen in agreement with predictions in K10 and Ventura et al. stellar evolution models. This is an important evidence supporting predictions of stellar evolution models for low-intermediate mass stars, which produce O-enriched stars a low metallicity. Such results are important to identify the best models for the chemical evolution of PNe from low metallicity stellar progenitors.

A full version of this work will be published elsewhere.

\section{Acknowledgements}

This work received financial support from DGAPA-PAPIIT (UNAM) grant IN109614.

\section{References}

Delgado-Inglada, G., Morisset, C., \& Stasinska, G. 2014, MNRAS, 440, 536

Evans, C. J., Bresolin, F., Urbaneja, M. A., Pietrzynski, G., \& Gieren, W., 2007, ApJ, 659, 1198 Karakas A. I. 2010, MNRAS, 403, 1413

Ott, J., Stilp, A. M., Warren, S. R. et al. 2012, AJ, 144, 123

Peña, M., Richer, M. G, \& Stasinska, G. 2007, A\&\&A, 144, 123

Peña, M., Stasinska, G., \& Richer, M. G. 2007, $A \& A$, 476, 745

Ventura, P., Di Criscienzo, M., Carini, R., \& D'Antona, F. 2013, MNRAS, 431,3642

Ventura, P., Di Criscienzo, M., D'Antona, F., et al. 2014, MNRAS, 437, 3274

Ventura, P., Dell'Agli, F., Schneider, R., et al. 2014, MNRAS, 439, 977 